PROCEEDINGS OF THE

AMERICAN MATHEMATICAL SOCIETY

Volume 127, Number 11, Pages 3393-3401

S 0002-9939(99)04936-9

Article electronically published on May 4, 1999

\title{
SINGULAR HYPERBOLIC SYSTEMS
}

\author{
C. A. MORALES, M. J. PACIFICO, AND E. R. PUJALS
}

(Communicated by Mary Rees)

\begin{abstract}
We construct a class of vector fields on 3-manifolds containing the hyperbolic ones and the geometric Lorenz attractor. Conversely, we shall prove that nonhyperbolic systems in this class resemble the Lorenz attractor: they have Lorenz-like singularities accumulated by periodic orbits and they cannot be approximated by flows with nonhyperbolic critical elements.
\end{abstract}

\section{INTRODUCTION AND STATEMENT OF RESULTS}

The geometric Lorenz attractor and the horseshoe are two basic examples in the modern theory of dynamical systems [11]. The latter is a classical example of a hyperbolic set and it was a main motivation to build up the hyperbolic theory [11]. The former was introduced in an attempt to model the Lorenz equation [5], [6]. These examples are different from the hyperbolic viewpoint: the horseshoe is a hyperbolic set and the Lorenz attractor is not. Still, the geometric Lorenz attractor resembles the horseshoe in some aspects: each is the closure of its periodic orbits, transitive and has sensitivity with respect to initial conditions.

The purpose of this paper is to introduce a class of vector fields in dimension three containing Axiom A systems and the geometric Lorenz attractor. This class will also contain the singular horseshoe, introduced in [9] to exhibit stable nonhyperbolic vector fields on 3-manifolds with boundary. Conversely, the nonhyperbolic elements in this class will resemble the Lorenz attractors or the singular horseshoes: they contain Lorenz-like singularities accumulated by periodic orbits and they cannot be approximated by flows with nonhyperbolic critical elements. The considered class will be introduced by imposing to a partially hyperbolic set to have volume expanding the central direction. The main motivation is [10] where it was proved that nontrivial $C^{1}$ robust transitive sets for a three-dimensional flow $X$ are partially hyperbolic with volume expanding central direction either for $X$ or $-X$.

To precisely state our results, let us recall some definitions and notations.

Let $M$ be a closed 3-manifold and let $\mathcal{X}^{r}(M)$ be the set of $C^{r}$ vector fields on $M$ endowed with the $C^{r}$ topology, $r \geq 1$. Given $X \in \mathcal{X}^{r}(M)$, the flow induced by $X$ is denoted by $X_{t}$. A compact set $\Lambda$, invariant by $X_{t}$, is transitive if it is the $\omega$-limit set of one of its orbits and isolated if there is an open set $U \supset \Lambda$ such that $\Lambda=\bigcap_{t \in \mathbb{R}} X_{t}(U)$. A set $U$ satisfying this property is an isolating block of $\Lambda$. An

Received by the editors November 24, 1997 and, in revised form, January 22, 1998.

1991 Mathematics Subject Classification. Primary 58F10, 58F15.

Key words and phrases. Lorenz attractor, hyperbolicity, Axiom A.

This work was partially supported by CNPq-Brasil, Faperj-Brasil, Pronex-Brasil. The first author was partially supported by CNRS-France. 
isolated compact transitive set $\Lambda$ of $X$ is $C^{r}$ robust transitive if there is an isolating block $U$ of $\Lambda$ such that $\bigcap_{t \in \mathbb{R}} Y_{t}(U)$ is transitive for every $Y C^{r}$-close to $X$. In what follows transitive sets are always nonempty and even nontrivial: it does not consist of a single critical element, i.e., either a singularity or a periodic orbit.

Recall that a compact invariant set $\Lambda \subset M$ is hyperbolic if there exists a continuous invariant splitting $T_{\Lambda} M=E^{u} \oplus E^{X} \oplus E^{s}$ such that $E^{X}$ is tangent to the flow $X_{t}$ and $E^{u}$ (resp. $E^{s}$ ) is expanded (resp. contracted) by the derivative $D X_{t}$. A basic set is a hyperbolic set which is either a single critical element or both isolated and transitive. A vector field $X$ is Axiom $A$ if its nonwandering set $\Omega(X)$ is both hyperbolic and the closure of its critical elements.

A compact invariant set $\Lambda$ of $X \in \mathcal{X}^{r}(M)$ is partially hyperbolic if there exists a continuous dominated splitting $T_{\Lambda} M=E^{s} \oplus E^{c u}$ such that $E^{c u}$ contains the direction of the flow and $E^{s}$ is one-dimensional and contracting. That is, there are constants $0<\lambda<1, c>0$ and $T>0$ such that

$$
\begin{gathered}
\left\|D X_{T} / E^{s}\right\| .\left\|D_{-T} / E^{c u}\right\|<c \lambda^{T} \text { (dominating condition), } \\
\left\|D X_{T} / E^{s}\right\| \leq c \lambda^{T} \text { (contracting condition). }
\end{gathered}
$$

Note that a nontrivial basic set is a partially hyperbolic set. The Lorenz attractors (either expanding or contracting [11]) are examples of partially hyperbolic sets which are not hyperbolic.

We say that the central direction $E^{c u}$ in a partially hyperbolic splitting $T_{\Lambda} M=$ $E^{s} \oplus E^{c u}$ is volume expanding if there are constants $T>0, k>1$ so that $\left|\operatorname{det}\left(D X_{T} / E^{c u}\right)\right|>k$. Partially hyperbolic sets with volume expanding central direction were considered independently in [12]. The term 'volume expanding' (used in [12]) looks better than 'surface expanding' due to possible higher dimensional versions of our results.

Definition 1. A compact invariant set $\Lambda$ of $X$ with singularities is singular hyperbolic if each of its singularities is hyperbolic and, either for $X$ or $-X, \Lambda$ is partially hyperbolic with volume expanding central direction. A singular basic set is a singular hyperbolic set which is both isolated and transitive.

As we already mentioned, it was proved that a compact $C^{1}$ robust transitive set with singularities is singular hyperbolic [10]. We shall see later that the expanding Lorenz attractor and the singular horseshoe are the prototype examples of singular basic sets which are not hyperbolic.

It is well known that a basic set is $C^{r}$ robust transitive and the closure of its critical elements (any $r$ ). However, a singular basic set could be neither $C^{r}$ robust transitive nor the closure of its critical elements (any $r$ ). The example is the one obtained by embedding the Cherry flow [3] as a singular hyperbolic set in $M$.

Definition 2. Let $\sigma$ be a singularity of a vector field $X$ contained in an invariant compact set $\Lambda$ of $X$. We say that $\sigma$ is attached to $\Lambda$ if $\sigma$ is accumulated by periodic orbits of $X$ in $\Lambda$. If $D X(\sigma)$ has real eigenvalues $\left\{-\lambda_{2},-\lambda_{3}, \lambda_{1}\right\}$, we say that $\sigma$ is Lorenz-like for $X$ when

$$
-\lambda_{2}<-\lambda_{3}<0<\lambda_{3}<\lambda_{1}
$$

Clearly, an attached singularity of $\Lambda$ is accumulated by regular (nonsingular) $X$-orbits in $\Lambda$. The converse is obviously false. In [4] it was proved that attached 
singularities cannot coexist with robustness of transitivity of $M$. Note that hyperbolic sets cannot have attached singularities.

There are singular hyperbolic sets which are also hyperbolic. The example (that is not transitive) is the union of a nontrivial basic set and a Lorenz-like singularity. Note that, by definition, a singular hyperbolic set must contain singularities. So, according to Definition 1, a suspended horseshoe [3] is partially hyperbolic but not singular hyperbolic. The contracting Lorenz attractor (see Chapter 7 in [11]) is also an example of a partially hyperbolic transitive set which is not singular hyperbolic.

Now, given a hyperbolic saddle-type singularity $\sigma$ of $X$ with real distinct eigenvalues, we denote by $W^{s s}(\sigma)$ (resp. $\left.W^{u u}(\sigma)\right)$ the strong stable (resp. unstable) manifold of $\sigma$.

It was proved in [10] that an attached singularity $\sigma$ of a $C^{1}$ robust transitive set $\Lambda$ of a vector field $X$ is Lorenz-like and satisfies

$$
\Lambda \cap W^{s s}(\sigma)=\{\sigma\},
$$

either for $X$ or $-X$ (see Figure 1). This was done as a first step in proving that those sets are singular hyperbolic.

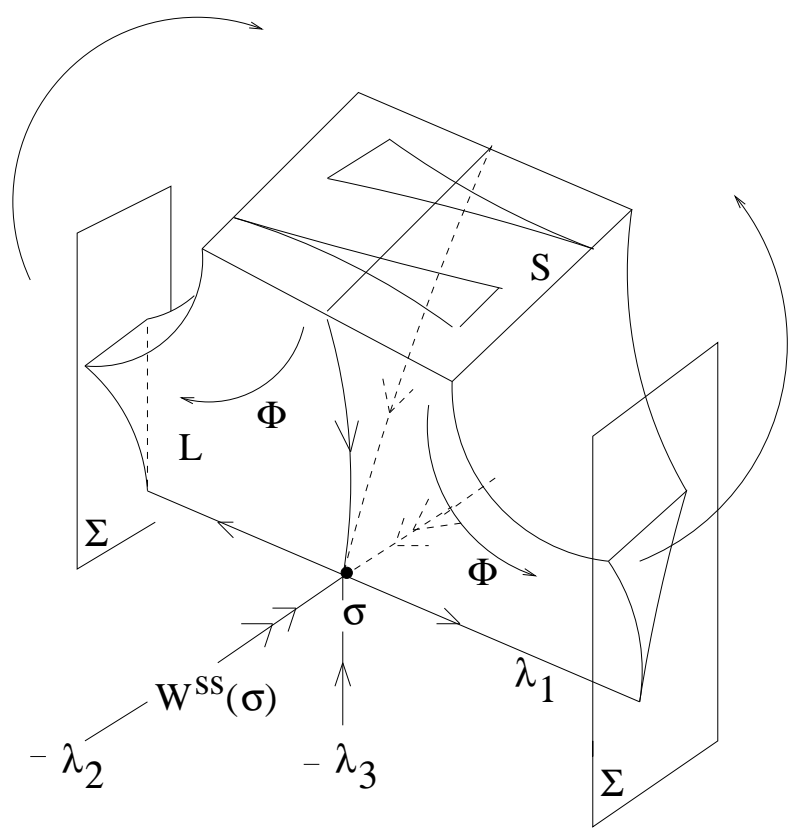

FiguRE 1

Such properties hold also for the geometric Lorenz attractor and the singular horseshoe (see [5] and [9]). In general we have

Theorem A. Let $\Lambda$ be a singular hyperbolic set of $X$ and assume that $\Lambda$ is nonhyperbolic. Then, $\Lambda$ has at least one singularity accumulated by regular $X$-orbits in $\Lambda$. In addition, the following holds either for $X$ or $-X$ : each attached singularity $\sigma$ of $\Lambda$ is Lorenz-like and satisfies

$$
\Lambda \cap W^{s s}(\sigma)=\{\sigma\} .
$$


It is known that if $X$ is an Axiom A vector field, then its nonwandering set is a disjoint union of basic sets [11]. This is the main motivation for the definition below.

Definition 3. A vector field $X \in \mathcal{X}^{r}(M)$ is a singular Axiom $A$ if

(1) the nonwandering set $\Omega(X)$ of $X$ is a disjoint union of basic or singular basic sets;

(2) $\Omega(X)$ is the closure of the critical elements of $X$.

Moreover, we say that a singular Axiom A satisfies the no-cycle condition (or has no cycles) if there is no set of regular orbits linking its basic or singular basic sets in a cyclic way. It follows easily from the definition that an Axiom A vector field (without cycles) is a singular Axiom A (without cycles). The following corollary is a simple consequence of Theorem A and Definition 3.

Corollary 1. Let $X$ be a singular Axiom $A$ vector field in $M$. Then,

(a) $X$ is Axiom $A$ if and only if there is no attached Lorenz-like singularities either for $X$ or $-X$;

(b) $X$ is $\Omega$-stable if and only if $X$ is Axiom A without cycles [11].

We shall describe in section 3 a singular Axiom A vector field without cycles and with a singular basic set equivalent to the Lorenz attractor ([5]). This example will belong also to the interior $\mathcal{G}^{r}(M)$ of the set of $C^{r}$ vector fields in $M$ whose critical elements are hyperbolic.

Now, there is a classification, in terms of hyperbolicity, of the $C^{1}$ interior of the set of diffeomorphisms in $M$ whose periodic points are hyperbolic: they are Axiom A without cycles (see [7]). The corresponding result for vector fields is not true because the Lorenz attractor is not a hyperbolic set. Our second result is the following.

Theorem B. A $C^{r}$ singular Axiom $A$ flow without cycles is in $\mathcal{G}^{r}(M)$ for any $r \geq 1$.

Notice that to be in $\mathcal{G}^{r}(M)$ is not sufficient to be singular Axiom A. Indeed, the singular cycle in [1] is in $\mathcal{G}^{1}(M)$ but it is not singular Axiom A for, in this case, the critical elements fail to be dense in the nonwandering set. However, such a singular cycle can be approximated by Axiom A flows without cycles. Indeed, we conjecture that singular Axiom A flows without cycles form an open and dense subset in $\mathcal{G}^{1}(M)$.

This paper is organized as follows. We prove our results at section 2. In section 3 we exhibit an example of a nonhyperbolic singular Axiom A flow with an invariant set topologically conjugate to the Lorenz attractor presented in [5].

\section{The PROOFS}

In this section we prove Theorems A and B. The basic tool will be the Poincaré flow associated to a compact invariant set without singularities. Let us recall this concept. Let $\Lambda \subset M$ be a compact invariant set of $X \in \mathcal{X}^{r}(M)$ without singularities. Set $N_{q}^{X}$ to be the orthogonal complement of the direction of the flow at $q \in \Lambda$. Given $v \in N_{q}^{X}$, define $P_{t}^{X, \Lambda}(v)$ as the orthogonal projection of $D X_{t}(v)$ on $N_{X_{t}(q)}^{X}$. The flow $P_{t}^{X, \Lambda}$ is the linear Poincaré flow of $X$ associated to $\Lambda$ (see [4]). Let $N_{\Lambda}^{X}=\bigcup_{q \in \Lambda} N_{q}$ be the normal subbundle of $X$ over $\Lambda$. The flow $P_{t}^{X, \Lambda}$ is 
hyperbolic if there is a continuous $P_{t}^{X, \Lambda}$-invariant splitting $N_{\Lambda}^{X}=G_{\Lambda}^{X} \oplus F_{\Lambda}^{X}$ such that $G_{\Lambda}^{X}$ is contracting and $F_{\Lambda}^{X}$ is expanding for $D P_{t}^{X, \Lambda}$.

It turns out that the hyperbolicity of a vector field without singularities can be expressed in terms of the hyperbolicity of the linear Poincaré flow associated to $X$. Indeed, the following result is proved in [4].

Lemma 1. A compact invariant set $\Lambda$ of $X$ without singularities is hyperbolic if and only if $P_{t}^{X, \Lambda}$ is hyperbolic.

It is clear that the linear Poincaré flow is not defined at a singularity. So there is no hope to characterize a singular hyperbolic set with singularities through the hyperbolicity of that flow. Despite this difficulty, we can still get some information from the restriction of the linear Poincare flow to $\Lambda^{*}=\Lambda \backslash \operatorname{Sing}_{X}(\Lambda)$, where $\operatorname{Sing}_{X}(\Lambda)$ is the set of singularities of $X$ in $\Lambda$. Let $\Lambda$ be a partially hyperbolic set of $X$ with $T_{\Lambda} M=E_{\Lambda}^{s} \oplus E_{\Lambda}^{c u}$ so that $E^{c u}$ is volume expanding. Set $E^{X}$ to be the flow direction of $X$ in $\Lambda$. Define the subbundle $F_{\Lambda^{*}}=N_{\Lambda^{*}} \cap E_{\Lambda^{*}}^{c u}$ over $\Lambda^{*}$. Clearly, $F_{\Lambda^{*}}$ is invariant by $P_{t}^{X, \Lambda^{*}}$. Given a linear operator $B$, let $m(B)$ be the minimum norm of $B$, that is, $m(B)=\left\|B^{-1}\right\|^{-1}$ when $B$ is invertible.

Lemma 2. Let $\Lambda$ be a partially hyperbolic set of $X$ whose central direction is volume expanding. Then, there are constants $\lambda, k>0$ such that

$$
m\left(P_{t}^{X, \Lambda^{*}} / F_{\Lambda^{*}}\right) \cdot m\left(D X_{t} / E_{\Lambda^{*}}^{X}\right) \geq k e^{\lambda t}, \forall t>0 .
$$

Proof. Let $T_{\Lambda} M=E^{s} \oplus E^{c u}$. As $\Lambda$ is compact and $E^{c u}$ is volume expanding we have that

$$
\left|\operatorname{det}\left(D X_{t} / E_{\Lambda}^{c}\right)\right| \geq k e^{\lambda t}, \forall t \geq 0
$$

(fixed $k, \lambda>0$ ). Moreover, $E_{\Lambda^{*}}^{c u}=F_{\Lambda^{*}} \oplus E_{\Lambda^{*}}^{X}$ by the definition of normal bundle.

Given $(v, u) \in F_{\Lambda^{*}} \oplus E_{\Lambda^{*}}^{X}$, let $R(v, u)$ be the parallelogram whose sides are $v$ and $u$. Set $\operatorname{vol}(R)$ the volume of $R(v, u)$. Then

$$
\operatorname{vol}(R)=\|v\| \cdot\|u\|
$$

In this case,

$$
\operatorname{vol}\left(D X_{t}(R)\right)=\left\|P_{t}^{X, \Lambda^{*}}(v)\right\| .\left\|D X_{t}(u)\right\|
$$

by definition of $P_{t}^{X, \Lambda^{*}}$. Thus, $\operatorname{vol}\left(D X_{t}(R)\right) \geq k e^{\lambda t}$ and the result follows.

Now we have

Lemma 3. Let $\Lambda$ be a singular hyperbolic set of $X$ and $\Lambda^{\prime} \subset \Lambda$ be a compact $X$ invariant set such that $\operatorname{Sing}_{X}\left(\Lambda^{\prime}\right)$ is empty. Then $\Lambda^{\prime}$ is hyperbolic. In particular, any critical element of $X$ in $\Lambda$ is hyperbolic.

Proof. Reversing the flow direction if necessary, we can assume that $\Lambda$ is partially hyperbolic for $X$. In this case, so does $\Lambda^{\prime}$. Let us prove that $\Lambda^{\prime}$ is hyperbolic. Assume that $T_{\Lambda^{\prime}} M=E^{s} \oplus E^{c u}$ with $E^{s}$ contracting and $E^{c u}$ volume expanding. So, all we have to prove is that $E^{c u}$ along $\Lambda^{\prime}$ splits in the flow direction together with an expanding bundle. For this, since $\Lambda^{\prime}$ has no singularities, we can consider the sub bundle $F=F_{\Lambda^{\prime}}$ defined as above. As $\Lambda^{\prime}$ is a compact partially hyperbolic set for $X$ without singularities, we have that $m\left(D X_{T} / E^{X}\right)$ is bounded and bounded away and below from zero. Thus, Lemma 2 implies that there exist positive constants $T$ and $\lambda<1$ so that $m\left(P_{t}^{X, \Lambda^{\prime}} / F_{\Lambda^{\prime}}\right) \geq k e^{\lambda T}$. This easily implies that $P_{T}^{X, \Lambda^{\prime}}$ is hyperbolic and applying Lemma 1 we conclude the proof. 
We point out that an isolated singular hyperbolic set can either implode or explode by small perturbations of the flow. Indeed, if we perturb the singular horseshoe, we can get both cases, depending on the way we do such a perturbation (see [1]). By explode we mean that for $Y$ near $X$, the nonwandering set of $Y$ restricted to $\bigcap_{t \in \mathbb{R}} Y_{t}(U)$ is a disjoint union of a finite number of transitive sets and this union contains at least two elements. By implode we mean that for $Y$ near $X$, such a nonwandering set is a finite number of critical elements of $Y$. The next result asserts that the new nonwandering set that arises after a perturbation of a singular hyperbolic set is still singular hyperbolic.

Proposition 1. Let $\Lambda$ be a singular hyperbolic set of $X \in \mathcal{X}^{r}(M), r \geq 1$. Then there exist a neighborhood $U$ of $\Lambda$ and a neighborhood $\mathcal{U} \subset \mathcal{X}^{r}(M)$ of $X$ such that if $Y \in \mathcal{U}$, then any invariant set of $Y$ in $U$ is singular hyperbolic.

Proof. As in the hyperbolic case, the existence of a continuous, invariant and dominated splitting for $\Lambda$ is an open property, that is, there are neighborhoods $U \supset \Lambda$ and $\mathcal{U} \supset X$ with the properties in the statement of the proposition. All we have to prove is that the central direction for $\Lambda_{Y}$, an invariant set in $U$ for $Y \in \mathcal{U}$ is volume expanding. And this is done following step by step Lemma 4.6 in [8], replacing expanding by volume expanding maps. We leave the details to the reader.

Proof of Theorem A. We prove the first part of Theorem A. Notice that $\operatorname{Sing}_{X}(\Lambda)$ $\neq \emptyset$ for otherwise $\Lambda$ is hyperbolic by Lemma 3. So, let $\sigma_{1} \in \operatorname{Sing}_{X}(\Lambda)$. If $\sigma_{1}$ is not in the closure of $\Lambda^{*}, \Lambda=\left\{\sigma_{1}\right\} \cup \Lambda_{1}$ (disjoint union) where $\Lambda_{1}$ is the closure of $\Lambda^{*}$. Since $\Lambda$ is nonhyperbolic, so is $\Lambda_{1}$. Moreover, $\operatorname{Sing}_{X}\left(\Lambda_{1}\right)$ is not empty for otherwise $\Lambda_{1}$ would be hyperbolic by Lemma 3 , a contradiction. Since $\operatorname{Sing}_{X}(\Lambda)$ is finite, repeating the process we finally reach a singularity in the closure of $\Lambda^{*}$ proving the result.

Now we prove the second part. Assume that $\Lambda$ exhibits a dominated splitting $T_{\Lambda} M=E^{s} \oplus E^{c u}$ where $E^{s}$ is contracting and $E^{c u}$ is volume expanding. Let $\sigma$ be a singularity of $X$ in $\Lambda$. Then $\sigma$ has at most two contracting eigenvalues. By contradiction, let us assume that $\sigma$ has just one contracting eigenvalue. Then $W^{s}(\sigma)$ is one-dimensional and since $\sigma$ is attached, at least one branch of this manifold (say $W_{r}^{s}$ ) is contained in $\Lambda$. Note that the central direction $E^{c u}$ contains the flow direction. Since any tangent vector at $q \in W_{r}^{s}$ which is not in the flow direction is eventually expanded by $D X_{t}$, we conclude that $E^{s}(q)=\{0\}$, giving a contradiction. This shows that $\sigma$ has exactly two contracting eigenvalues. It follows also that that the eigenvalues of $\sigma$ must be real. Indeed, if this were not the case, $\sigma$ would be an attracting singularity, which contradicts the fact that $\sigma \in \Lambda$, with $\Lambda$ being partially hyperbolic with $E^{c u}$ volume expanding. As the splitting over $\Lambda$ at $\sigma$ coincides with the splitting given by the eigenvalues of $\sigma$ at $\sigma$, the volume expanding condition at $E^{c u}$ also implies that $\sigma$ is Lorenz-like for $X$. In particular, $\operatorname{dim}\left(W^{s}(\sigma)\right)=2$. In this case, let us prove that $\Lambda \cap W^{s s}(\sigma)=\sigma$. Suppose that this is not true. As $W^{s s}(\sigma)$ is one-dimensional, it follows that $\Lambda$ contains one separatrix of $W^{s s}(\sigma)$ (say $W_{l}$ ). But the central direction must contain the flow direction and so it is tangent to $W_{l}$ at any $q \in W_{l}$. Moreover, the central direction is also contained into the stable plane generated by the contracting eigenvalues of $\sigma$. Otherwise the angle between $E_{q}^{s}$ and $E_{q}^{c u}$ goes to zero when $q \in W_{l}$ approaches $\sigma$. Then the central direction $E^{c u}$ contains $E^{s}$, a contradiction. 
Proof of Theorem B. Let $X$ be a singular Axiom A flow and $\Omega(X)=\Lambda_{1} \cup \ldots \cup \Lambda_{n}$ its nonwandering set. Now, by Proposition 1, there are neighborhoods $U_{i}$ of $\Lambda$ and $\mathcal{U}$ of $X$ of such that any compact invariant set of $Y \in \mathcal{U}$ in $U_{i}$ is singular hyperbolic. Then, any critical element of $Y$ in $U_{i}$ is hyperbolic by Lemma 3.

Now we use the fact that $\Omega(X)$ has a finite decomposition in basic or singular basic sets together with the fact that $\Omega(X)$ has noncycles to obtain a filtration for $\Omega(X)$ and, as in the hyperbolic case, we conclude the proof (see [2]).

\section{An example}

In this section we present a singular Axiom A vector field in $\mathcal{G}^{r}(M)$ with a singular basic set $L$ equivalent to the geometric Lorenz attractor introduced in [5]. For this, we give a description of $L$ and, afterward, we extend $L$ to the whole manifold $M$.

To start, consider the linear differential equation in $\mathbb{R}^{3}$ given by:

$$
L\left\{\begin{array}{l}
\dot{x}=\lambda_{1} x, \\
\dot{y}=-\lambda_{2} y, \\
\dot{z}=-\lambda_{3} z .
\end{array}\right.
$$

See Figure 1. The origin $\sigma=(0,0,0)$ is a hyperbolic saddle singularity of $L$ with eigenvalues $\left\{-\lambda_{2},-\lambda_{3}, \lambda_{1}\right\}$. We assume that $-\lambda_{2}<-\lambda_{3}<0<\lambda_{3}<\lambda_{1}$ and so $\sigma$ is Lorenz-like (see section 1 ).

The flow induced by $L$ is given by

$$
L_{t}(x, y, z)=\left(x e^{\lambda_{1} t}, y e^{-\lambda_{2} t}, z e^{-\lambda_{3} t}\right) .
$$

Notice that the lines $(0, y, 0)$ (denoted by $\hat{y}),(x, 0,0)$ and $(0,0, z)$ are preserved by $L_{t}$, any $t \geq 0$. In particular, the line $\hat{y}$ and the plane $(x, 0, z)$ (denoted by $\hat{x z}$ ) are invariant by $L_{t}$.

Now, there exists a first return map $\Phi$ from the cross section $S=\{z=1\}$ into $\Sigma=\{x= \pm 1\}$. Actually $\Phi$ is only defined on $\bar{S}=S \backslash\{(0, y, 1)\}$.

For any $q \in \bar{S}$, let $t(q)>0$ be the first positive time along the orbit of $q$ so that $L_{t(q)} \in S$. Then $\Phi(q)=L_{t(q)}(q)$. Define

$$
\bar{R}=\left\{\left(L_{t}(q), t\right):(q, t) \in \bar{S} \times[0, t(q)]\right\}
$$

and

$$
R=\bar{R} \cup W_{l o c}^{s}(\sigma) \cup W_{l o c}^{u}(\sigma) .
$$

Here $W_{l o c}^{l}(\sigma)$ is the local stable (resp. unstable) manifold associated to $\sigma, l=s, u$ (see [11]). It turns out that $R$ is a linear region which is pinched along $W_{l o c}^{u}(\sigma)$. Let $f: \Sigma \rightarrow S$ be a homeomorphism such that it has a $C^{\infty}$ extension to small neighborhoods of both connected components of $\Sigma$. We identify in $R,(\Phi(q), t(q))$ with $(f(\Phi(q), t(q)), 0)$ for $q \in \bar{S}$ and $q \in W_{l o c}^{u}(\sigma) \cap \Sigma$ with $(f(q), 0)$.

With this identification we obtain a new manifold $\mathcal{L}_{f}$ and a flow acting on it. We call this system the singular suspension of $\Phi$, by analogy with the suspension of smooth maps ([3]).

The topological structure of $\mathcal{L}_{f}$ is known. It is not a smooth manifold due to the pinched region at the local unstable manifold, but it can be embedded in $\mathbb{R}^{3}$ 
if $f$ is orientation preserving. This is enough for our proposes. Now, the linear vector field (2) lifts to a vector field in $\mathcal{L}_{f}$, which we still denote by $L$. Actually, $L$ is the lift of a positive semiflow whose dynamics is related to the map $f$. We have the following properties with respect to the natural coordinate system $(x, y, z)$ in $\mathcal{L}=\mathcal{L}_{f}$ :

(i) $L$ preserves the fibration $\mathbb{R}^{3}=E \oplus F$, where $E=\hat{y}$ and $F=\hat{x z}$;

(ii) the flow of $L$ is given by (2).

Observe that although we can choose $f$ in a way that $L$ satisfies (i) above, it is not possible to choose it such that $L$ preserves neither the line $(x, 0,0)$ nor the line $(0,0, z)$, due to obvious topological obstructions.

We claim that $L$ is a singular hyperbolic set. In fact, one has

$$
\left\|D L_{t} / E\right\|=e^{-\lambda_{2} t}
$$

and

$$
D L_{t} / F=\left(\begin{array}{cc}
e^{\lambda_{1} t} & 0 \\
0 & e^{-\lambda_{3} t}
\end{array}\right)
$$

So $E$ is contracting and $F$ is volume expanding since $\left|\operatorname{det}\left(D L_{t} / F\right)\right|=e^{\left(\lambda_{1}-\lambda_{3}\right) t}$, with $\lambda_{1}-\lambda_{3}>0$. We also have that $E$ dominates $F$. Indeed, since $m\left(D L_{t} / F\right) \geq$ $e^{-\lambda_{3} t}$ we have that

$$
\frac{\left\|D L_{t} / E\right\|}{m\left(D L_{t} / F\right)} \leq e^{\left(\lambda_{3}-\lambda_{2}\right) t}
$$

This proves that the maximal invariant set of $L$ in $\mathcal{L}$ is singular hyperbolic and completes the proof.

\section{REFERENCES}

[1] R. Bamón, R. Labarca, R. Mañé, M.J. Pacifico, The explosion of singular cycles, Publ. Math. IHES 78 (1993), 207-232. MR 94m:58152

[2] C. Conley, Isolated sets and the Morse index, CBMS Regional Conf. Series in Math., Amer. Math. Soc. Providence R.I. 38 (1978). MR 80c:58009

[3] W. de Melo, J. Palis, Geometric theory of dynamical systems, Springer-Verlag (1982). MR 84a:58004

[4] C. I. Doering, C. I., Persistently transitive vector fields on three-dimensional manifolds, Dynamical Systems and Bifurcation Theory, Pitman Research Notes in Mathematics Series 160 (1987), 59-89. MR 89c:58111

[5] J. Guckenheimer, A strange strange attractor, The Hopf bifurcation and its applications, Applied Mathematical Series 19 (1976), 368-381. MR 58:13209

[6] J. Guckenheimer, R. F. Williams, Structural stability of Lorenz attractors, Publ. Math. IHES 50 (1979), 59-72. MR 82b:58055a

[7] S. Hayashi, Diffeomorphisms in $\mathcal{F}^{1}$ satisfy Axiom A, Erg. Th. \& Dyn. Sys. 12 (1992), 233253. MR 94d:58081

[8] M. Hirsch, C. C. Pugh, Stable manifolds and hyperbolic sets, Proc. Amer. Math. Soc. Symp. Pure Math. 9 (1970), 133-163. MR 42:6872

[9] R. Labarca, M. J. Pacífico, Stability of singular horseshoe, Topology 25 (1986), 337-352. MR 87h:58106

[10] C.A. Morales, M.J. Pacifico, E.R. Pujals, On $C^{1}$-robust transitive sets for three-dimensional flows, C. R. Acad. Sci. Paris 326 (1998), 81-86. CMP 99:02 
[11] J. Palis, F. Takens, Hyperbolicity and sensitive chaotic dynamic at homoclinic bifurcation, Cambridge University Press 35. MR 94h:58129

[12] L.P. Shilnikov, Talk at Nizhny-Novgorov University (1996).

(C. A. Morales) Université de Bourgogne, Laboratoire de Topologie, B.P.400, 21011, Dijon CEDeX-France

E-mail address: cmorales@u-bourgogne.fr

Current address: Instituto de Matemàtica, Universidade Federal do Rio de Janeiro, C.P. 68.530, CEP 21.945-970, Rio de Janeiro, Brazil

E-mail address: morales@impa.br

(M. J. Pacifico and E. R. Pujals) Instituto de Matemàtica, Universidade Federal do Rio De Janeiro, C. P. 68.530, CEP 21.945-970, Rio de Janeiro, BraziL

E-mail address: pacifico@impa.br

E-mail address: enrique@impa.br 